\title{
Twin Jet Effects on Noise of Round and Rectangular Jets: Experiment and Model
}

\author{
Rick Bozak ${ }^{1}$ \\ NASA Glenn Research Center, Cleveland, OH, 44135
}

\begin{abstract}
Many subsonic and supersonic aircraft concepts proposed by NASA's Fundamental Aeronautics Program have asymmetric, integrated propulsion systems. The asymmetries in the exhaust of these propulsion systems create an asymmetric acoustic field. The asymmetries investigated in the current study are from twin jets and rectangular nozzles. Each effect produces its own variation of the acoustic field. An empirical model was developed to predict the acoustic field variation from round twin jets with twin jet spacing from 2.6 to 5.6, where $s$ is the center-to-center spacing over the jet diameter. The model includes parameters to account for the effects of twin jet spacing, jet static temperature ratio, flight Mach number, frequency, and observer angle (both polar and azimuthal angles). The model was then applied to twin 2:1 and 8:1 aspect ratio nozzles to determine the impact of jet aspect ratio. For the round and rectangular jets, the use of the model reduces the average magnitude of the error over all frequencies, observation angles, and jet spacings by approximately $0.5 \mathrm{~dB}$ when compared against the assumption of adding two jets incoherently.
\end{abstract}

\section{Nomenclature}

$S \quad=$ Non-dimensional twin jet spacing, center-to-center spacing/nozzle diameter

$D \quad=$ Nozzle diameter, inches

$U_{j} \quad=$ Ideally-expanded jet velocity, inches per second

$f \quad=$ Frequency, $\mathrm{Hz}$

St $\quad=$ Strouhal number, $f D / U_{j}$

$\theta \quad=$ Polar angle, from the inlet axis, degrees

$\varphi \quad=$ Azimuthal angle relative to the twin jet symmetry plane, degrees

$M_{a} \quad=$ Acoustic Mach number

$M_{f} \quad=$ Flight Mach number

$T_{S r} \quad=$ Static temperature ratio

$S P L=$ Sound pressure level, $\mathrm{dB}$ ref. $20 \mu \mathrm{Pa}$

$\triangle S P L \quad=$ Twin jet effect, $\mathrm{dB}$

$\triangle S P L_{r m s} \quad=$ Average magnitude of twin jet effect, $\mathrm{dB}$

$w \quad=$ Model for the twin jet effect, $\mathrm{dB}$

$\Delta w \quad=$ Model error, $\mathrm{dB}$

$\Delta w_{r m s} \quad=$ Average magnitude of model error, $\mathrm{dB}$

\section{Introduction}

Tntegrated propulsion systems are utilized in many of the aircraft concepts being evaluated by NASA's IFundamental Aeronautics Program. Some of these integrated propulsion systems contain multiple asymmetric exhausts. These exhaust systems exhibit an asymmetric acoustic field that can be difficult to predict. Experiments were performed to isolate the effects of twin jets on the radiated acoustic field. Once the effects were isolated, they were parameterized into an empirical model to predict the asymmetric sound field produced by twin jets.

Twin jet aeroacoustic experiments have been performed at NASA's Langley ${ }^{1}$ and at Glenn ${ }^{2,3}$ Research Centers. These experiments investigated the impact of jet spacing, jet conditions, flight Mach number, non-parallel jets, and a hybrid wing-body shield. Both far-field and phased array acoustic measurements were made to determine the

\footnotetext{
${ }^{1}$ Research Aerospace Engineer, Acoustic Branch, NASA Glenn Research Center, AIAA Member.
} 
impact on radiated noise. Recently, additional experiments investigated the impact of rectangular nozzles on the twin jet effect ${ }^{4}$.

While experiments have quantified the twin jet effect, it can be difficult to determine the impact of twin jets on the noise radiated from an aircraft. Therefore, an effort was undertaken to incorporate an empirical model into the second generation of the Aircraft NOise Prediction Program (ANOPP2) ${ }^{5}$. ANOPP2 incorporates the ability to add the asymmetry in the radiated acoustic field that the twin jet effect exhibits. The approach is similar to that of Veltin, et al. ${ }^{6}$ where a delta correction factor is applied to single jet predictions to obtain a twin jet prediction. An empirical model is developed from round twin jet data to account for the acoustic impact of the twin jet effect. This model incorporates factors to account for parameters with the largest impact on the twin jet effect. The model is then evaluated against far-field acoustic data from round and rectangular twin jet experiments.

\section{Experimental Dataset}

The data were obtained from experiments conducted on the High Flow Jet Exit Rig (HFJER) in the AeroAcoustic Propulsion Laboratory (AAPL) at NASA Glenn Research Center. The HFJER is mounted in the Nozzle Acoustic Test Rig (NATR) which provides simulated flight around the nozzle ${ }^{7}$. A schematic of the twin jet model is shown in Fig. 1. The y-duct divides the core flow of the dual stream HFJER into two streams. The internal pressure taps shown at the inlet to the s-ducts monitor the pressure in each leg of the twin jet model to ensure equal flow through each nozzle. The s-ducts following the y-duct are used to vary the spacing between the nozzles. The straight adapter ensures parallel flow from the jets. Fig. 2 shows the installation of the twin jet model mounted on the HFJER in the AAPL. Round 2-inch diameter convergent conical nozzles (TCON), as well as 2:1 (A2Z0) and 8:1 (A8Z0) aspect ratio 2.13-inch area equivalent diameter rectangular nozzles were tested. Three twin jet nozzle spacings were evaluated to determine the sensitivity of spacing on the radiated noise. These spacings are shown in Table 1 as a ratio of the center-to-center spacing to the nozzle diameter, $s$. The rectangular nozzles were tested with their major axis aligning with the plane containing both jets. The closest spacing obtained with the 8:1 aspect ratio rectangular nozzles was $s=3.32$ due to an interference between the nozzles.
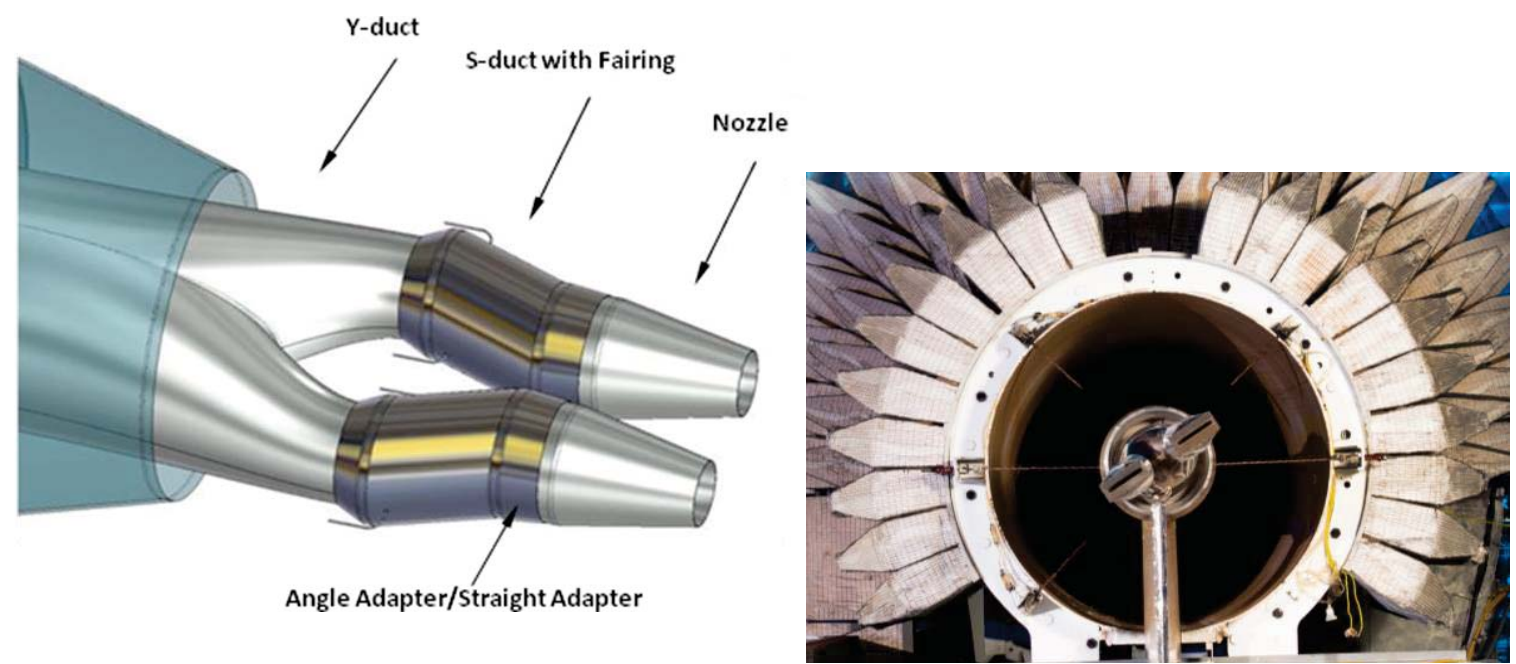

Figure 1. Twin jet model components.

Figure 2. Twin jet model mounted on the Nozzle Acoustic Test Rig (NATR).

Table 1. Round and rectangular twin jet spacings, center to center distance over diameter, $s$.

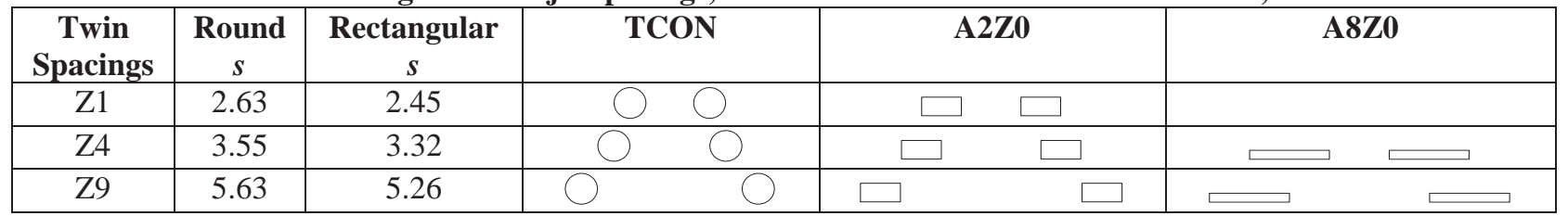




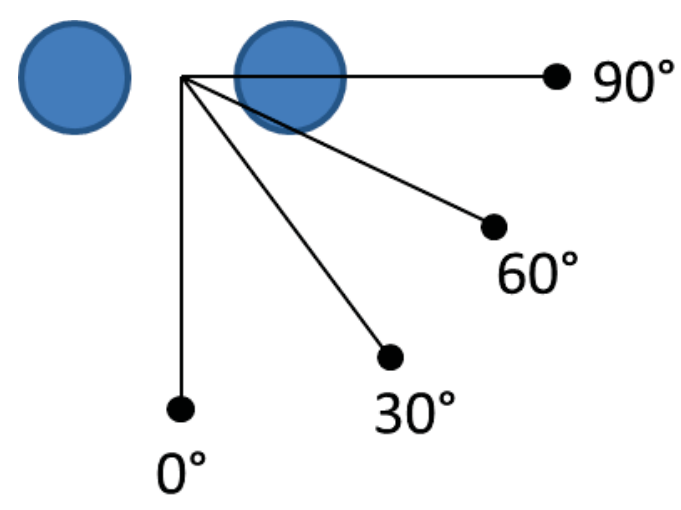

Figure 3. Azimuthal $(\varphi)$ microphone measurement locations.

The acoustic data were measured in the AAPL by 24 microphones on a 45 -foot polar arc from $45^{\circ}$ to $160^{\circ}$ relative to the NATR inlet. By rotating the twin model relative to the microphone array, data were acquired at the four azimuthal angles in Fig. 3. The microphone measurements were corrected for microphone response, and for the free stream shear layer and atmospheric absorption ${ }^{8,9}$. The jet operating conditions used in the experiments spanned a variety of conditions that were intended to isolate the twin effects from jet temperature, acoustic Mach number, and flight Mach number. Jet acoustic Mach numbers $M_{a}$ from 0.5 to 1.33, nozzle static temperature ratios $T_{s r}$ from 0.84 to 2.27, and free stream Mach numbers $M_{f}$ of $0,0.1,0.2$ and 0.25 were used in the experiments. The data used for modeling were obtained with the eight jet conditions shown in Table 2.

Perhaps the simplest expected effect of having twin jets relative to a single jet is to expect the sound power to be the incoherent addition of two identical single jet spectra (i.e. single jet plus $3 \mathrm{~dB}$ ). This will be the baseline for considering the effect of twin jet spectral directivity. The twin jet effect in sound pressure level $(\triangle S P L)$ is defined as the sound pressure level for the twin jets $\left(S P L_{t w i n}\right)$ minus the sound pressure level of a single jet at the same conditions $\left(S P L_{\text {single }}\right)$ plus $3 \mathrm{~dB}$. Thus, the twin jet effect is

$$
\triangle S P L=S P L_{T w i n}-\left(S P L_{\text {Single }}+3\right)
$$

When considering the twin jet effect for non-axisymmetric nozzles that have an azimuthally varying sound field, the twin jet effect is calculated by subtracting the sound pressure level of a single non-axisymmetric nozzle plus 3dB from the sound pressure level of the same types of nozzles at the same observer location. Since the twin jet effect does not vary rapidly with frequency, it will be modeled on a 1/3-octave frequency scale. The twin jet effect has been shown to vary from $-3 \mathrm{~dB}$ to $+3 \mathrm{~dB}$ depending on observer location and frequency ${ }^{2}$. This effect translates to as much as \pm 3 EPNdB depending on the twin jet configuration ${ }^{3}$.

The twin jet effect is shown in Fig. 4 for round nozzles at an acoustic Mach number $M_{a}=1.33$, a static temperature ratio $T_{s r}=1.76$, and a flight Mach number $M_{f}=0.10$. In the figure each contour plot shows the variation of the twin jet effect $\triangle S P L$ over polar angle $\theta$ and Strouhal number St. The contour plots are arranged with a row for each of the four azimuthal angles $\varphi$, and columns for each of the twin jet spacings $s$. The color contours range from $3 \mathrm{~dB}$ (blue) to $+3 \mathrm{~dB}$ (red). The largest reductions in noise are seen at an azimuthal angle $\varphi$ of $90^{\circ}$ and the largest increase is seen at $\varphi=0^{\circ}$. The $\triangle S P L$ smoothly varies between the minimum and maximum as $\varphi$ goes from $90^{\circ}$ to $0^{\circ}$ and are centered about $\triangle S P L=0$ when all polar and azimuthal angles are combined. The majority of the twin jet effect is concentrated at large polar angles and higher frequencies. Both the magnitude and frequency dependence of the twin jet effect change with jet spacing.

As a measure of the relative magnitude of the twin jet effect, the root-mean-square (rms) of $\triangle S P L$ over frequency, polar and azimuthal angles, and over jet spacing was calculated for each set of nozzles and at each jet condition.

$$
\Delta S P L_{r m s}=\sqrt{\int_{S} \int_{\varphi} \int_{\theta} \int_{S t}\left[S P L_{T w i n}-\left(S P L_{\text {Single }}+3\right)\right]^{2}} d S t d \theta d \varphi d s
$$

The relative magnitude of the twin jet effect $\triangle S P L_{r m s}$ can also be interpreted as a measure of the error in assuming that the twin jet effect is simply to double the sound of a single jet.

The tabulated values of $\triangle S P L_{r m s}$ for each case are given in the middle three columns of Table 2. The tabulated values show that the twin jet effect increases with increasing jet static temperature ratio $T_{s r}$ from $0.9 \mathrm{~dB}$ to $1.4 \mathrm{~dB}$.

3

American Institute of Aeronautics and Astronautics 
The magnitude of the twin jet effect decreases with increasing flight Mach number $M_{f}$. Furthermore, while these observations are consistent between the round nozzles and the 2:1 rectangular nozzles, the magnitude of the twin jet effect is relatively independent of jet condition for the 8:1 rectangular nozzles. The empirical modeling effort to be described next will focus on the most significant parameters: frequency, polar angle, azimuthal angle, jet spacing, jet temperature, and flight Mach number.
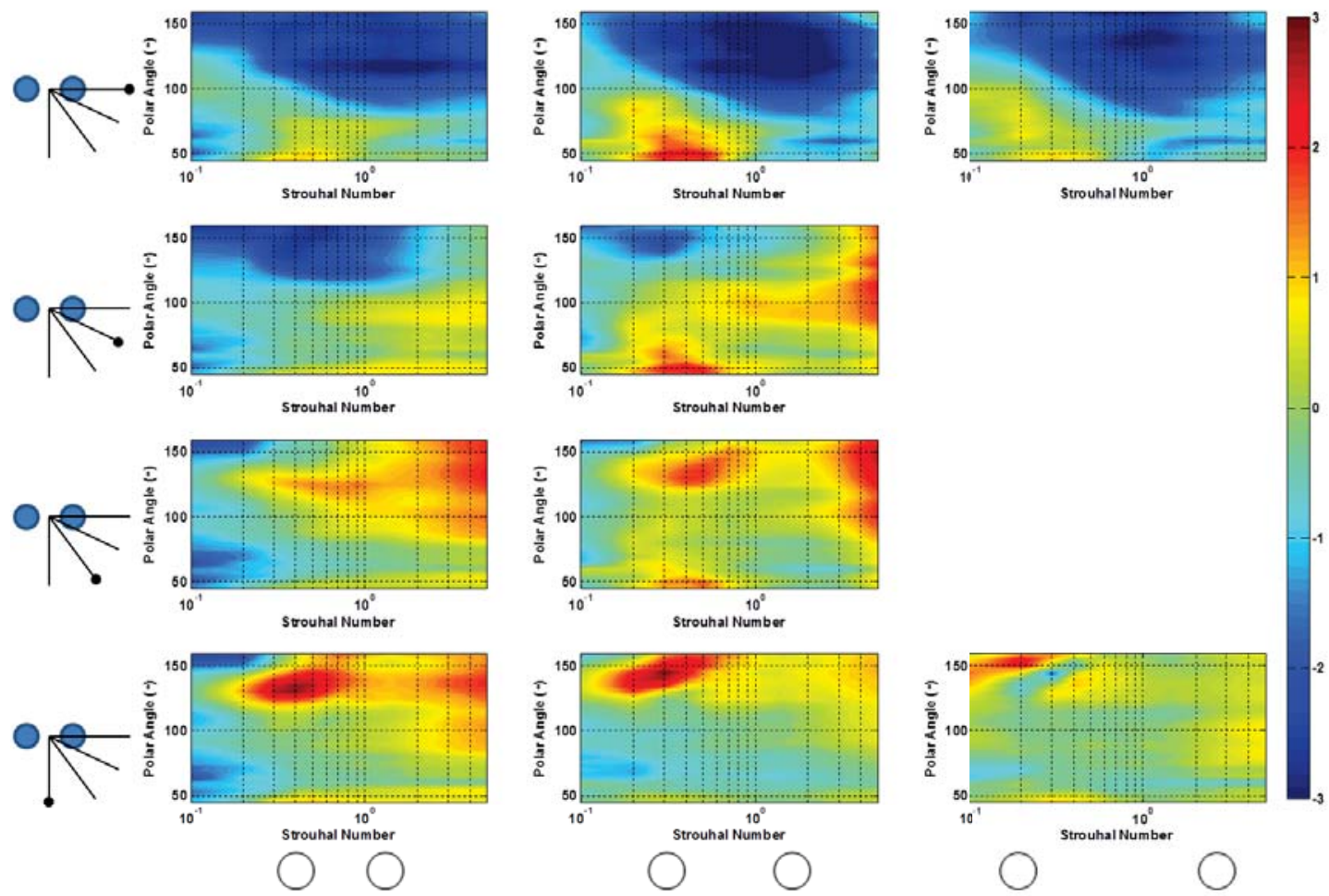

Figure 4. The twin jet effect $\triangle S P L$ for $M_{a}=1.33, T_{s r}=1.76, M_{f}=0.10$ with the four azimuthal angles, shown in Fig. 3, in the vertical direction and three jet spacings, shown in Table 1, in the horizontal direction.

Table 2. Root-mean-square of the Twin Jet Effect, $\Delta S P L_{r m s}$, and Model Errors, $\Delta w_{r m s}$ in dB over all polar and azimuthal angles, and frequencies for each nozzle tested (columns) and at each jet condition (rows).

\begin{tabular}{|c|c|c|c|c|c|c|c|c|}
\hline \multicolumn{3}{|c|}{ Jet Conditions } & \multicolumn{3}{c|}{$\begin{array}{c}\text { Magnitude of the Twin Jet } \\
\text { Effect }\left(\boldsymbol{\Delta S P L}_{\boldsymbol{r m s}}\right)(\mathbf{d B})\end{array}$} & \multicolumn{3}{c|}{$\begin{array}{c}\text { Magnitude of the Model } \\
\text { Error }\left(\boldsymbol{\Delta} \boldsymbol{w}_{\boldsymbol{r m s}}\right)(\mathbf{d B})\end{array}$} \\
\hline $\boldsymbol{M}_{\boldsymbol{a}}$ & $\boldsymbol{T}_{\boldsymbol{s r}}$ & $\boldsymbol{M}_{\boldsymbol{f}}$ & $\mathbf{T C O N}$ & $\mathbf{A 2 Z 0}$ & $\mathbf{A 8 Z 0}$ & $\mathbf{T C O N}$ & $\mathbf{A 2 Z 0}$ & $\mathbf{A 8 Z 0}$ \\
\hline 0.70 & 0.90 & 0 & 0.9 & 0.9 & 1.4 & 0.5 & 0.7 & 1.2 \\
\hline 0.90 & 0.84 & 0 & 1.1 & 1.0 & 1.4 & 0.7 & 0.7 & 1.1 \\
\hline 0.90 & 1.76 & 0 & 1.1 & 1.3 & 1.3 & 0.5 & 0.6 & 0.8 \\
\hline 1.33 & 2.27 & 0 & 1.4 & 1.5 & 1.5 & 0.6 & 0.7 & 1.0 \\
\hline 1.33 & 1.76 & 0 & 1.4 & 1.4 & 1.4 & 0.7 & 0.6 & 0.8 \\
\hline 1.33 & 1.76 & 0.10 & 1.2 & 1.3 & 1.4 & 0.6 & 0.7 & 0.8 \\
\hline 1.33 & 1.76 & 0.20 & 1.1 & 1.1 & 1.3 & 0.8 & 1.0 & 1.0 \\
\hline 1.33 & 1.76 & 0.25 & 1.0 & 1.3 & 1.4 & 1.0 & 1.5 & 1.5 \\
\hline
\end{tabular}




\section{Empirical Modeling Methodology}

If the twin jet effect was estimated by adding two incoherent jets, the error could be $\pm 3 \mathrm{~dB}$ at certain frequencies and observation angles. The root-mean-square values of $\triangle S P L_{r m s}$ in the middle three columns of Table 2 show that the average magnitude of the twin jet effect varies from 1 to $1.5 \mathrm{~dB}$ depending on the jet operating conditions. To reduce this error, an empirical model is proposed. The model incorporates factors to account for twin jet parameters which have been observed to have the largest effect.

At a specific jet condition, the sound pressure level of a single round jet is dependent on Strouhal number $S t$ and polar observer angle $\theta$. The twin jet effect $\triangle S P L$ is dependent on these parameters as well as azimuthal angle $\varphi$, the non-dimensional twin jet spacing $s$, jet static temperature ratio $T_{s r}$, and flight Mach number $M_{f}$. The model $w$ for the twin jet effect is broken up into separate additive effects for spacing $w_{s}$, temperature $w_{T}$, and flight Mach number $w_{F}$, as shown in Eq. (3).

$$
w\left(s, T_{s r}, M_{f}, S t, \theta, \varphi\right)=w_{s}(s, S t, \theta, \varphi)+w_{T}\left(T_{s r}, s, S t, \theta, \varphi\right)+w_{F}\left(M_{f}, s, S t, \theta, \varphi\right)
$$

The effect of spacing was modeled with a quadratic polynomial, where the effect becomes zero as spacing goes to zero, as shown below in Eq. (4). This condition ensures that as the spacings decrease towards zero, the twin jet effect becomes the incoherent addition of two identical jets. This condition allows the model to be used for twin jet spacings less than 2.6; however, the model should not be used for twin jet spacings above 6 since the model was based on twin jet spacings up to 5.6 and continues to grow unbounded with increased spacing. A regression to this polynomial gave two coefficients; $w_{\mathrm{s} 1}$ and $w_{\mathrm{s} 2}$. These coefficients were determined for each combination of Strouhal number, polar angle, and azimuthal angle for the first five jet conditions in Table 2. These jet conditions provide a distribution of acoustic Mach numbers and jet static temperature ratios. Since data were not acquired at the twin jet spacing of 5.625 for azimuthal angles of $30^{\circ}$ and $60^{\circ}$, the $w_{\mathrm{s} 2}$ coefficient was set to zero at these locations.

$$
w_{s}(s, S t, \theta, \varphi)=w_{s 2}(S t, \theta, \varphi) s^{2}+w_{s 1}(S t, \theta, \varphi) s
$$

The effect of jet temperature was modeled in terms of jet static temperature ratio $T_{s r}$. The ability of a shear layer to reflect acoustic waves is dependent on the ratio of acoustic impedances between the two fluids. This ratio for a jet shear layer simplifies to the square-root of the jet static temperature ratio. The coefficients $w_{\text {T0 }}$ for the jet temperature effect $\left(w_{T}\right)$, shown below in Eq. (5), were determined from the first five jet conditions in Table 2 for each combination of Strouhal number, polar angle, and azimuthal angle. Including the product of the nondimensional spacing $s$ ensures that the model for the twin jet effect $w$ is 0 when the spacing is 0 .

$$
w_{T}\left(T_{s r}, s, S t, \theta, \varphi\right)=w_{T 0}(S t, \theta, \varphi) \sqrt{T_{s r}} s
$$

The effect of flight Mach number was modeled as a linear effect, as shown in Eq. (6). The last four conditions in Table 2 were used to determine a coefficient $w_{\mathrm{F} 0}$ for each combination of Strouhal number, polar angle, and azimuthal angle. Including the product of the non-dimensional spacing again ensures that the model for the twin jet effect $w$ is 0 when the spacing is 0 .

$$
w_{F}\left(M_{F}, s, S t, \theta, \varphi\right)=w_{F 0}(S t, \theta, \varphi) M_{f} S
$$

The empirical model development described provides a predicted twin jet effect based on twin jet spacing, static temperature ratio and flight Mach number with four coefficients that vary with Strouhal number, polar angle, and azimuthal angle. Since the twin jet effect smoothly varies over frequency, polar angle, and azimuthal angle, interpolation can be used to obtain results for other frequencies and angles.

\section{Evaluation of the Empirical Model}

The twin jet empirical model is comprised of four coefficients for each combination of $1 / 3$-octave frequency, polar angle, and azimuthal angle where data were acquired. While this results in about 2700 coefficients, the calculation of the twin jet effect is simple following the equations described above. The model is evaluated by comparing predictions to experimental data at each of the jet conditions shown in Table 2.

The modeling error in the twin jet effect $\Delta w$ is calculated by subtracting the model prediction $w$, from the data $\triangle S P L$. Or put another way, it is the difference between the new prediction for twin jet sound, $S P L_{\text {Single }}+3+w$, and the data $S P L_{T w i n}$ 


$$
\Delta w=S P L_{T w i n}-\left(S P L_{\text {Single }}+3+w\right)
$$

Therefore, positive errors show that the model under-predicted the twin jet effect and negative errors show that the model over-predicted the effect. These errors will be evaluated for each set of nozzles over the range of frequencies, observation angles, spacings, and jet conditions. Similar to the definition of $\triangle S P L_{r m s}$ used above to measure the error of a simple 'add 3dB' model, $\Delta w_{r m s}$ is defined in Eq. (8) as the root-mean-square of the modeling error:

$$
\Delta w_{r m s}=\sqrt{\int_{s} \int_{\varphi} \int_{\theta} \int_{S t}\left[S P L_{\text {Twin }}-\left(S P L_{\text {Single }}+3+w\right)\right]^{2}} d S t d \theta d \varphi d s
$$

The differences in error between the simple model for twin jet noise $\Delta S P L_{r m s}$ and empirical model $\Delta w_{r m s}$, given in Table 2, quantify the improvement that the empirical model provides relative to the simple incoherent addition model (i. e. adding 3dB).

\section{A. Round Twin Jets}

The model error $\Delta w$ for twin jets at an acoustic Mach number of 1.33, static temperature ratio of 1.76, and flight Mach number of 0.1 is shown in Fig. 5. This jet condition corresponds to the twin jet effect shown in Fig. 4. The error is within $\pm 1 \mathrm{~dB}$ in the plane of the jets $\left(\varphi=90^{\circ}\right)$, but increases to $\pm 2 \mathrm{~dB}$ perpendicular to the plane of the jets $(\varphi$ $\left.=0^{\circ}\right)$. The $\pm 2 \mathrm{~dB}$ errors are concentrated downstream near the location of peak single jet noise $\left(\theta>140^{\circ}\right)$. For these jet conditions, the average magnitude of the model error reduced from $1.4 \mathrm{~dB}\left(\triangle S P L_{r m s}\right)$ with the simple model to $0.7 \mathrm{~dB}\left(\Delta w_{r m s}\right)$, as shown in Table 2. To see how well the model works at different temperatures Fig. 6 shows the model error $\Delta w$ for an unheated static jet at an acoustic Mach number of 0.9. The maximum errors are approximately $\pm 1.5 \mathrm{~dB}$. The positive error is concentrated at a polar angle $(\theta)$ of $90^{\circ}$ and azimuthal angle $(\varphi)$ of $90^{\circ}$. The magnitude of the model error is reduced from about $1 \mathrm{~dB}\left(\triangle S P L_{r m s}\right)$ to about $0.6 \mathrm{~dB}\left(\Delta w_{r m s}\right)$ for the unheated jet conditions in Table 2. And finally, to illustrate the sensitivity of the model to flight, in Fig. 7 the model error $\Delta w$ is shown at an acoustic Mach number of 1.33, static temperature ratio of 1.76, and flight Mach number of 0.2. The error increases with increasing spacing up to $\pm 3 \mathrm{~dB}$. The maximum and minimum error is concentrated in the peak single jet noise direction $\left(\theta>140^{\circ}\right)$ perpendicular to the plane containing both jets $\left(\varphi=0^{\circ}\right)$ for the widest jet spacing. The magnitude of the error is not significantly reduced by applying the twin jet model $w$ for jet conditions with flight. The factor accounting for flight Mach number in Eq. (6), is not sufficient at the highest flight Mach number.

\section{B. Rectangular Twin Jets}

The twin jet model $w$ based on data from round jets was applied to data from the 2:1 and 8:1 aspect ratio rectangular jets. Since azimuthal variation in noise radiated from a rectangular jet will be accounted for separately by using the spectral directivity of a single rectangular jet, the twin jet effect is isolated from the rectangular effect. Table 2 shows that the magnitude of the twin jet effect $\triangle S P L_{r m s}$ is increasing as aspect ratio increases. For the rectangular jets, measurements were only obtained at $\varphi=0^{\circ}$ and $90^{\circ}$. Fig 8 shows the model error $\Delta w$ for the 2:1 aspect ratio rectangular nozzle at an acoustic Mach number of 1.33 and static temperature ratio of 1.76 without flight. The errors are about $\pm 1 \mathrm{~dB}$ in downstream polar angles at an azimuthal angle of $0^{\circ}$. Table 2 shows that the improvements in the model error are consistent with those of the twin round nozzles. Fig. 9 shows model error $\Delta w$ for the 8:1 aspect ratio rectangular nozzle at an acoustic Mach number of 1.33 and static temperature ratio of 1.76 without flight. The error spans from $+0.5 \mathrm{~dB}$ to $-3 \mathrm{~dB}$ implying that the model over-predicted the twin jet effect. Table 2 shows that while the twin jet effect increases with increasing jet aspect ratio, the model error also increases with increasing jet aspect ratio, meaning that the effects of aspect ratio and jet by jet shielding are definitely not independent at this high value of aspect ratio. While the error is increasing with jet aspect ratio, the application of the twin jet model based on round data to the 8:1 aspect ratio rectangular nozzle averages $0.4 \mathrm{~dB}$ reduction in the magnitude of the error over the range of jet conditions in Table 2. 

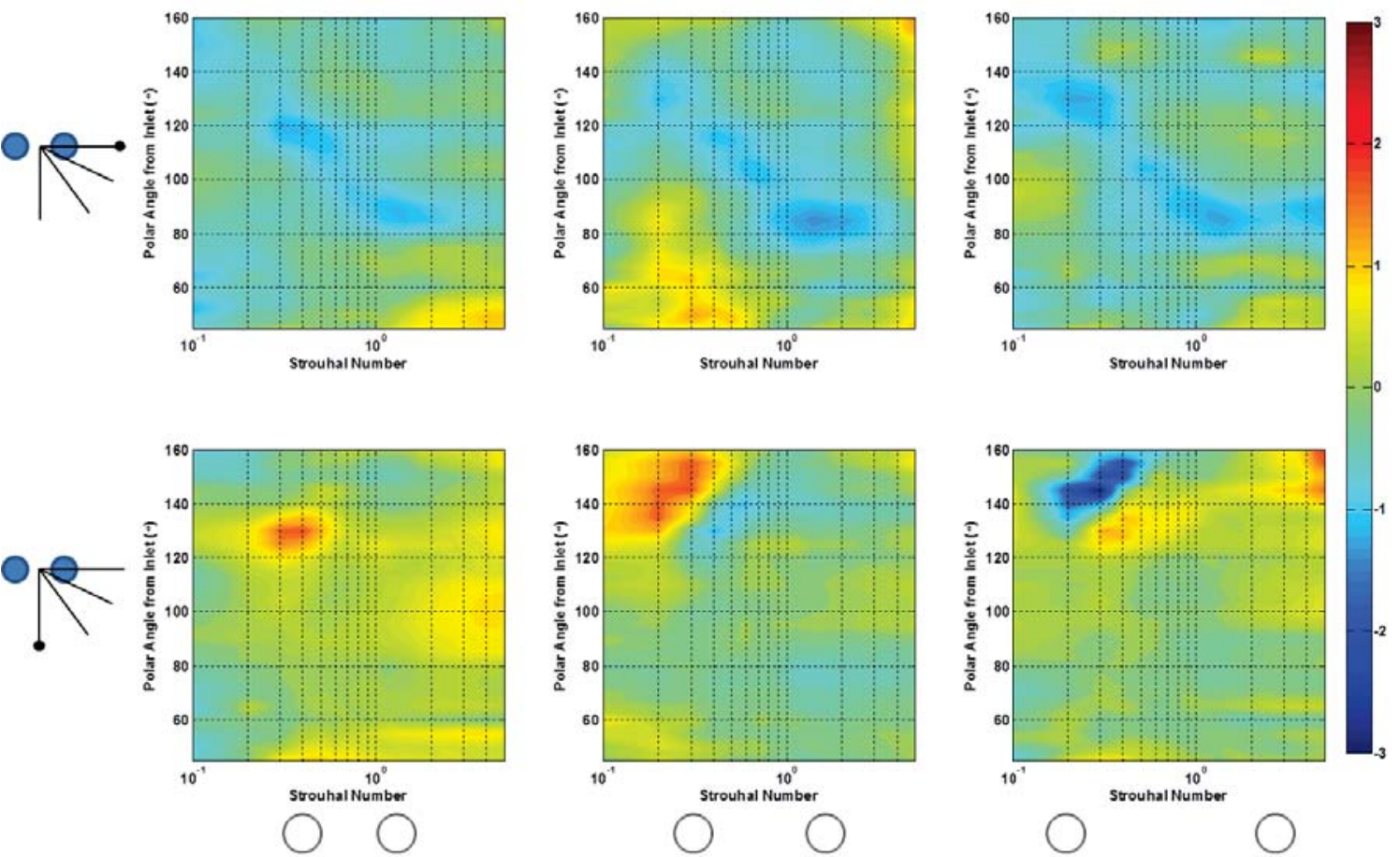

Figure 5. Model error $\Delta w$ when applying the model to round jets at $M_{a}=1.33, T_{s r}=1.76, M_{f}=0.10$. Observer azimuthal angle in the plane of the nozzles (top) and out of plane (bottom), jet spacings $s=2.63$ (left), 3.55 (center) and 5.63 (right).
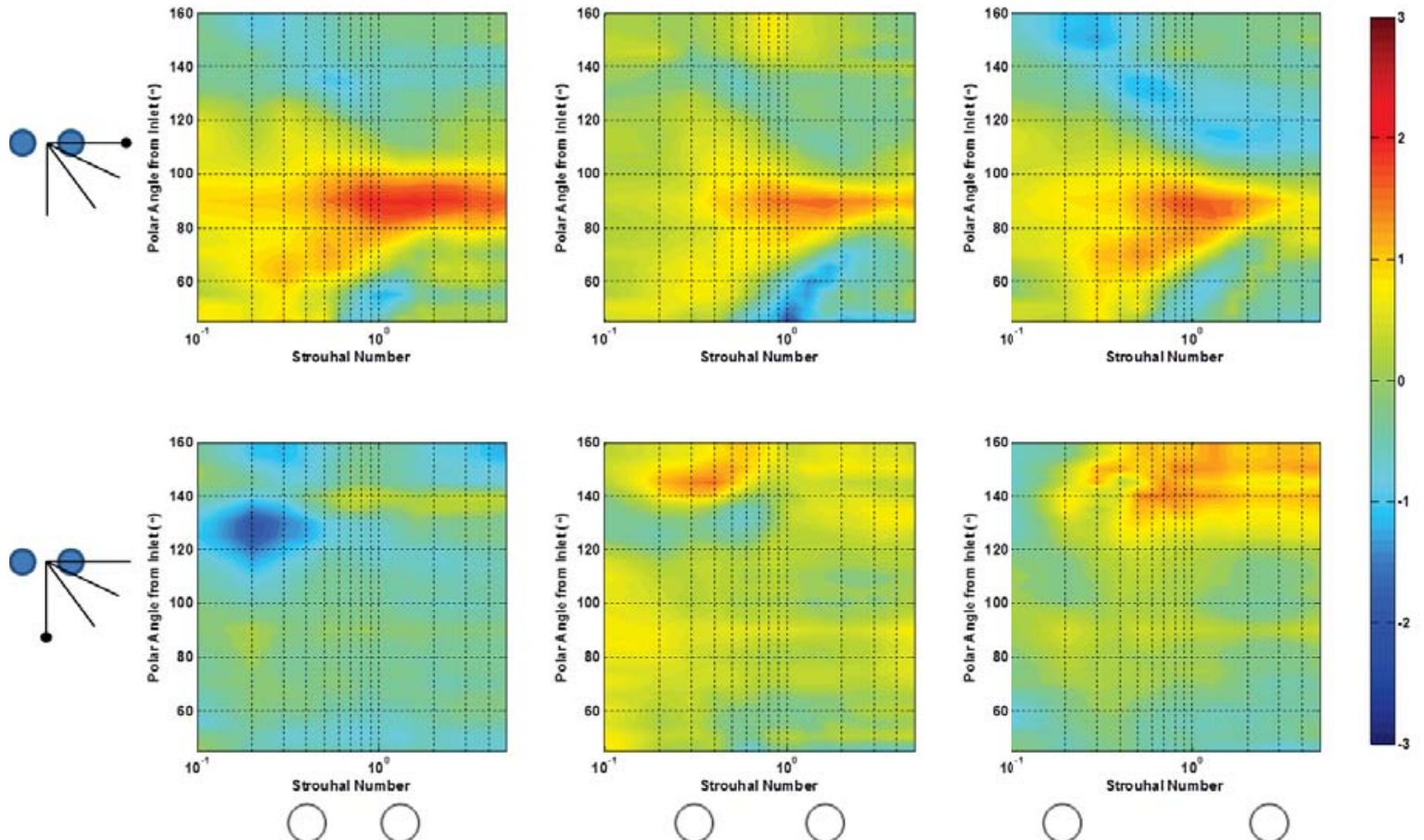

Figure 6. Model error $\Delta w$ when applying the model to round jets at $M_{a}=0.9$, cold, $M_{f}=0$. Observer azimuthal angle in the plane of the nozzles (top) and out of plane (bottom), jet spacings $s=2.63$ (left), 3.55 (center) and 5.63 (right). 

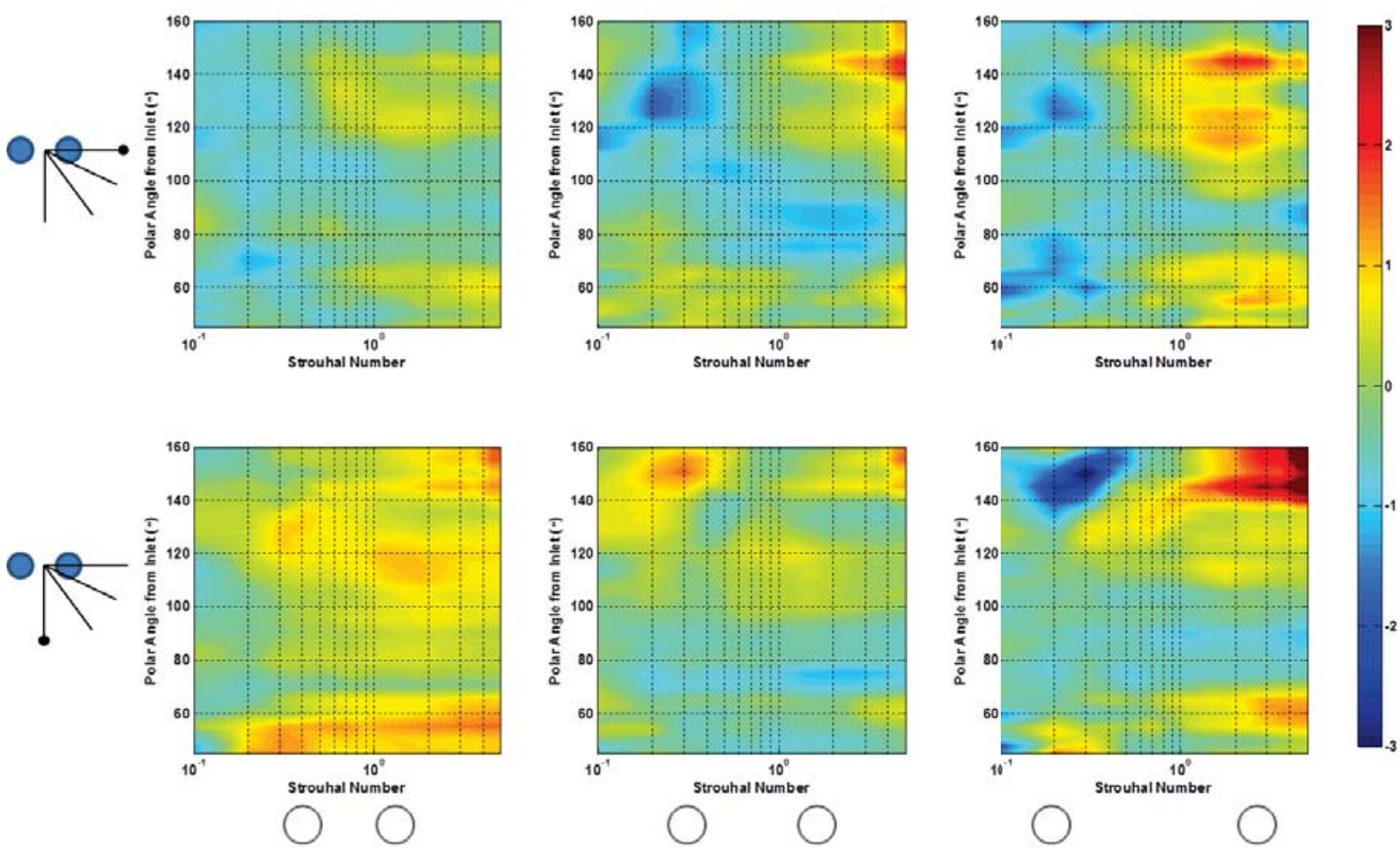

Figure 7. Model error $\Delta w$ for round jets at $M_{a}=1.33, T_{s r}=1.76, M_{f}=0.20$. Observer azimuthal angle in the plane of the nozzles (top) and out of plane (bottom), jet spacings $s=2.63$ (left), 3.55 (center) and 5.63 (right).
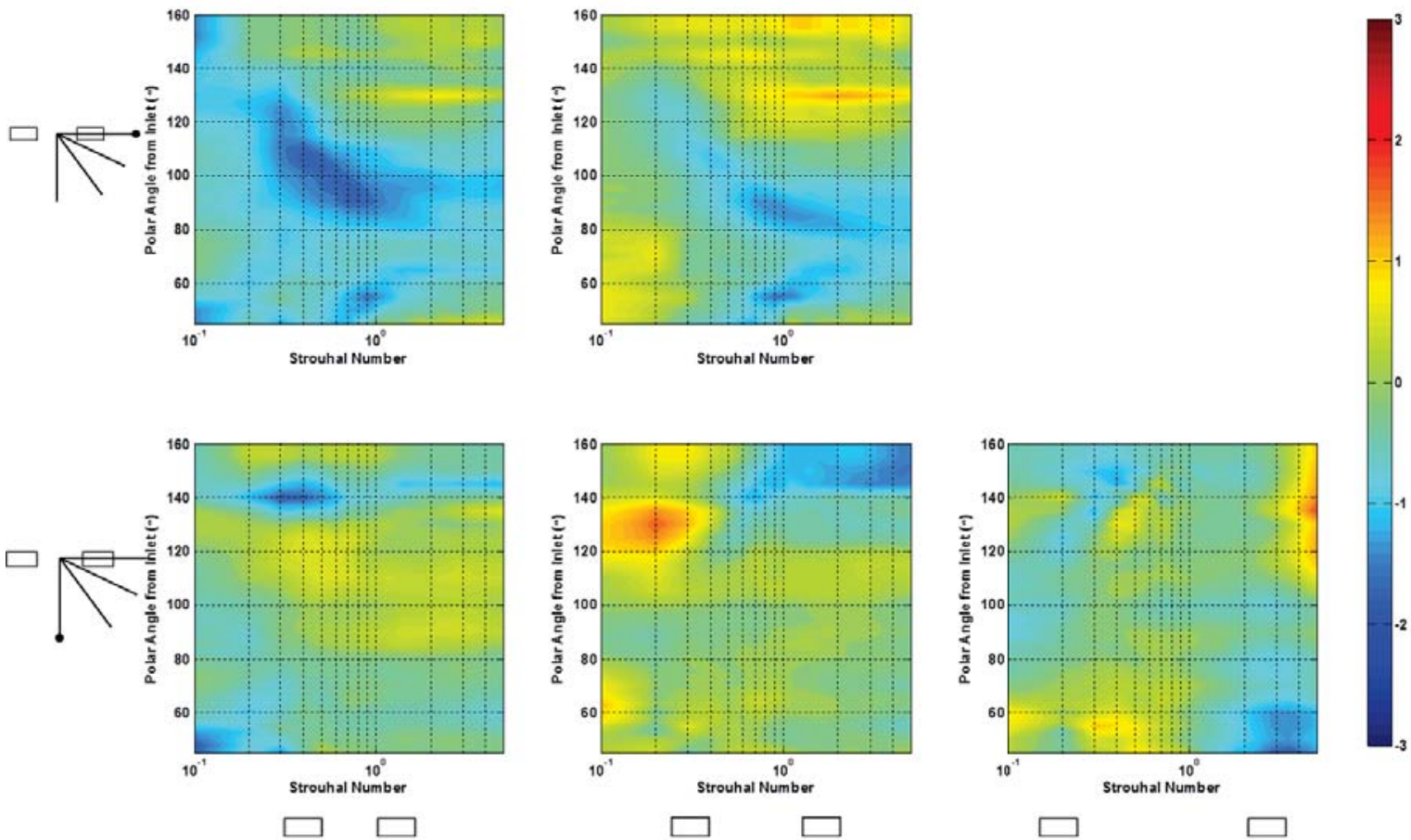

Figure 8. Model error $\Delta w$ when applying the model to predict the sound pressure levels of a 2:1 rectangular nozzle at $M_{a}=1.33, T_{s r}=1.76, M_{f}=0$. Observer azimuthal angle in the plane of the nozzles (top) and out of plane (bottom), jet spacings $s=2.45$ (left), 3.32 (center) and 5.26 (right). 

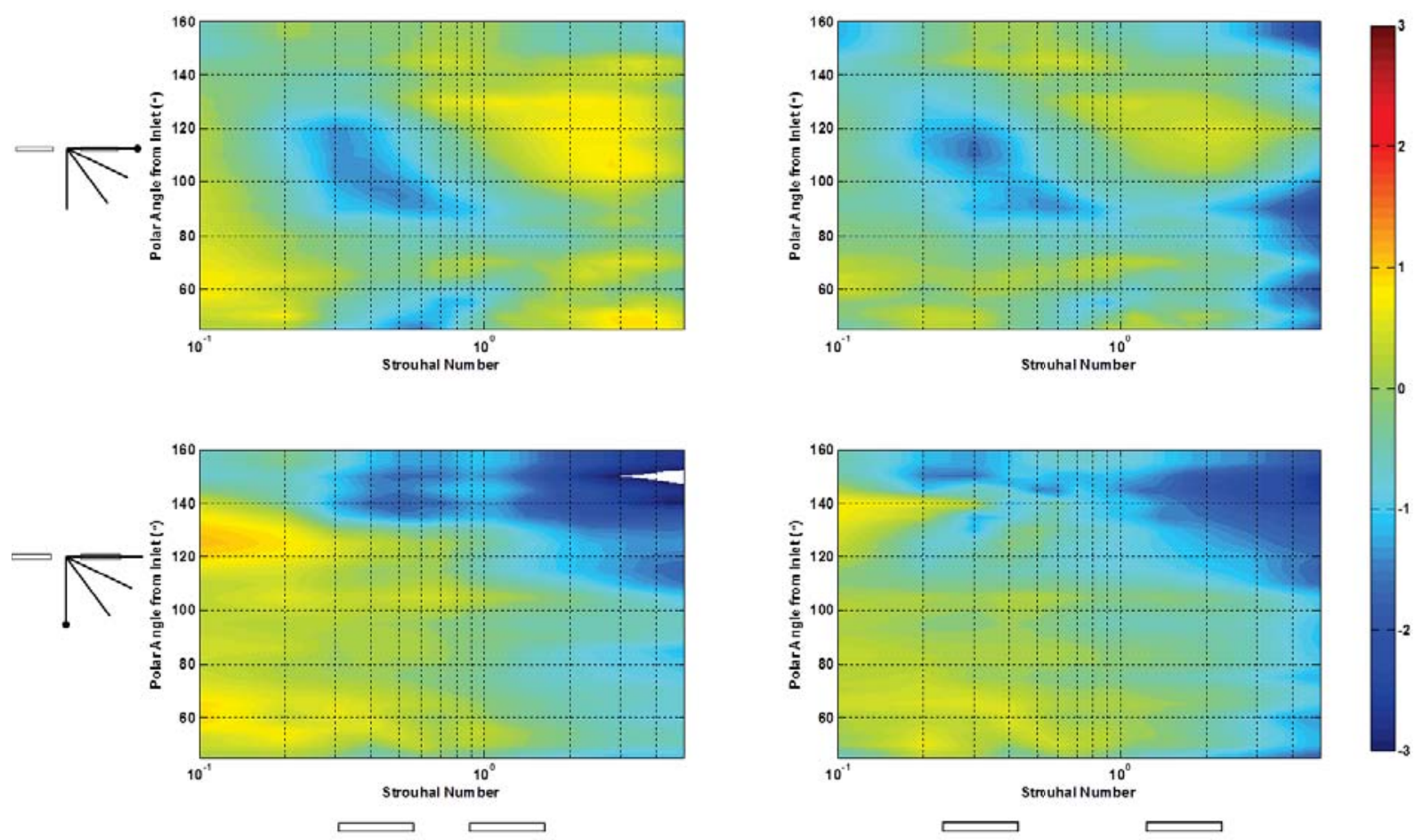

Figure 9. Model error $\Delta w$ when applying the model to predict the sound pressure levels of an 8:1 rectangular nozzle at $M_{a}=1.33, T_{s r}=1.76, M_{f}=0$. Observer azimuthal angle in the plane of the nozzles (top) and out of plane (bottom), jet spacings $s=3.32$ (left) and 5.26 (right).

\section{Conclusions}

Acoustic experiments were performed on twin round and rectangular jets to investigate the impact of nozzle spacing, jet temperature and Mach number, flight speed, and nozzle aspect ratio. The twin round jet data from this test were used to create an empirical model that accounts for twin jet variations over frequency, observer angles, jet spacing, jet static temperature ratio, and flight Mach number beyond the simplistic doubling of the sound levels. This empirical model was then exercised over a range of jet conditions and compared against data for both round and rectangular nozzles. The largest errors exist at the highest flight Mach numbers. For the round jets as well as the 2:1 aspect ratio rectangular nozzles, the use of the model reduces the average magnitude of the error by approximately $0.5 \mathrm{~dB}$ when compared against the assumption of adding two jets incoherently. The reduction in error for the $8: 1$ aspect ratio nozzles is only about $0.4 \mathrm{~dB}$ as the twin jet effect is not as independent of aspect ratio by this high value.

\section{Acknowledgments}

This work was supported by the High Speed Project of NASA’s Fundamental Aeronautics Program. 


\section{References}

${ }^{1}$ Doty, M., J., "Investigation of Twin Jet Aeroacoustic Properties near a Hybrid Wing-Body Shield," AIAA Journal, 1-13 , April 15, 2014. doi: 10.2514/1.J052783.

${ }^{2}$ Bozak, R., and Henderson, B., "Aeroacoustic Experiments with Twin Jets," NASA Glenn Research Center, AIAA-2011-2790, Portland, OR, Jun. 2011.

${ }^{3}$ Bozak, R., "The Impact of Subsonic Twin Jets on Airport Noise," NASA Glenn Research Center, AIAA-2012-2299, Colorado Springs, CO, Jun. 2012.

${ }^{4}$ Bridges, J., Brown, C., and Bozak, R., "Experiments on Exhaust Noise of Tightly Integrated Propulsion Systems," NASA Glenn Research Center, AIAA-2014-2904, Atlanta, GA, Jun. 2014.

${ }^{5}$ Lopes, L. V., and Burley, C., L., "Design of the Next Generation Aircraft Noise Prediction Program: ANOPP2," AIAA2011-2854, Portland, OR, Jun. 2011.

${ }^{6}$ Veltin, J., McLaughlin, D. K., and Morris, P. J., "Improvement of Acoustic Models for Community Noise Exposure Prediction," AIAA-2008-12, Reno, NV, Jan. 2008.

${ }^{7}$ Soeder, R. H., Wnuk, S. P., and Loew, R. A., "Nozzle Acoustic Test Rig User Manual,” NASA TM 2006-212939, 2006.

${ }^{8}$ Amiet, R. K., "Correction of Open Jet Wind Tunnel Measurements for Shear Layer Refraction," United Aircraft Research Laboratories, AIAA 75-532, Hampton, VA, Mar. 1975.

${ }^{9}$ ANSI S1.26-1995 (R2004), "Method for the Calculation of the Absorption of Sound by the Atmosphere." 